\title{
Photoproduction of Wide-Angle Electron-Positron Pairs at High Energies
}

\author{
J. G. Asbury*, William K. Bertram,* U. Becker, P. Joos, M. Rohde, and A. J. S. Smith* \\ Deutsches Elektronen-Synchrotron DESY, Hamburg, Germany \\ AND \\ S. Friedlander, C. L. Jordan, and Samuel C. C. Ting \\ Department of Physics, Columbia University, New York, New York $\dagger$
}

(Received 2 February 1967)

\begin{abstract}
Using the DESY 6.2-GeV electron synchrotron, we have measured the photoproduction on carbon of electron-positron pairs, for a range in the invariant pair mass of $150-550 \mathrm{MeV}$. To detect the pairs and to discriminate against the intense pion background, a magnetic spectrometer, counters, and fast electronics were used. The results are in agreement with the predictions of first-order quantum electrodynamics, in contradiction with earlier experiments. A comparison of our results with other tests of quantum electrodynamics at small distances is discussed.
\end{abstract}

\section{INTRODUCTION}

$\mathrm{R}^{\mathrm{E}}$ ECENT experiments at Cambridge Electron Accelerator (CEA $)^{1}$ and Cornell $^{2}$ indicate a disagreement with the rate predicted by first-order quantum electrodynamics (QED) for the production of electronpositron pairs by high-energy photons. The deviation occurs in two forms. First, in the published CEA result the absolute electron-positron rate at low-momentum transfers (normalization) does not agree with QED. Second, in both experiments the ratio of the experimental rate to the theoretical rate increases with increasing momentum transfer.

In an experiment performed at the DESY $6.2-\mathrm{GeV}$ electron synchrotron ${ }^{3}$ we have measured the yield of wide-angle $e^{+} e^{-}$pairs produced in the reaction

$$
\gamma+\text { carbon } \rightarrow e^{+}+e^{-}+\text {carbon. }
$$

The results show that first-order QED correctly predicts the $e^{+} e^{-}$pair yield for momentum transfers to the virtual electron up to $400 \mathrm{MeV} / c$, in contradiction to the above earlier experiments.

The photoproduction of electron-positron pairs is given, to first order in a perturbation-theory expansion, by the three Feynman graphs shown in Fig. 1. The first

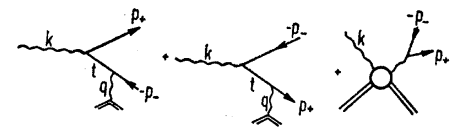

FIG. 1. Feynman diagrams for electron-positron pair production.

* Volkswagen Foundation Fellow at DESY.

† This work, a DESY-Columbia collaboration, was supported in part by the U. S. Atomic Energy Commission.

${ }^{1}$ R. B. Blumenthal, D. C. Ehn, W. L. Faissler, P. M. Joseph, L. J. Lanzerotti, F. M. Pipkin, and D. G. Stairs, Phys. Rev. 144, 1199 (1966). (This paper includes a complete discussion of the literature concerning pair production by photons.)

2 R. M. Talman, Bull. Am. Phys. Soc. 11, 380 (1966).

3 J. G. Asbury, W. K. Bertram, U. Becker, P. Joos, M. Rohde, A. J. S. Smith, S. Friedlander, C. L. Jordan, and C. C. Ting, Phys. Rev. Letters 18, 65 (1967).

${ }_{4}^{4}$ J. D. Bjorken, S. D. Drell, and S. C. Frautschi, Phys. Rev. 112,1409 (1958). In the text this reference is referred to as BDF. two, the Bethe-Heitler (BH) graphs, have been calculated by Bjorken, Drell, and Frautschi (BDF),${ }^{4}$ who included the effects of recoil of the target nucleus and of elastic nuclear form factors, and arrived at the following expression for the laboratory cross section:

$$
d \sigma_{B \mathrm{H}}=\frac{\alpha^{3} M}{4 \pi^{2}} \frac{d^{3} p_{+}}{E_{+}} \frac{d^{3} p_{-}}{E_{-}} \frac{\lambda_{11}(K)}{p_{+} \cdot Q+p_{-} \cdot Q-p_{+} \cdot p_{-}},
$$

where

$$
\begin{aligned}
& \lambda_{11}(K)=\frac{1}{2 q^{4}} \mathfrak{F}_{1}\left(q^{2}\right)\left\{\frac{m^{2} q^{2}}{(K \cdot p)^{2}}\right. \\
& \left.-2\left[\frac{K \cdot p_{+}}{K \cdot p_{-}}+\frac{K \cdot p_{-}}{K \cdot p_{+}}+\frac{q^{2} p_{+} \cdot p_{-}}{K \cdot p_{+} K \cdot p_{-}}\right]\right\}+\frac{1}{2 q^{4}} \Im_{2}\left(q^{2}\right) \\
& \times\left\{\frac{2 m^{2}\left(p_{+} \cdot P\right)^{2}}{\left(K \cdot p_{-}\right)^{2}}-\frac{q^{2}\left[\left(p_{+} \cdot P\right)^{2}+\left(p_{-} \cdot P\right)^{2}\right]}{K \cdot p_{+} K \cdot p_{-}}\right\}
\end{aligned}
$$

and

$$
K=\frac{p_{+} \cdot Q+p_{-} \cdot Q-p_{+} \cdot p_{-}}{M-E_{-}\left(1-\cos \theta_{-}\right)-E_{+}\left(1-\cos \theta_{+}\right)},
$$

and where the form factors $\mathfrak{F}_{1}\left(q^{2}\right)$ and $\mathfrak{F}_{2}\left(q^{2}\right)$ are related to the charge and magnetic form factors $G_{E}\left(q^{2}\right)$ and $G_{M}\left(q^{2}\right)$ by

$$
\begin{aligned}
& \left.\mathscr{F}_{1}\left(q^{2}\right)=G_{E}{ }^{2}+\left(q^{2} / 4 M^{2}\right) G_{M^{2}}{ }^{2}\right], \\
& \mathscr{F}_{2}\left(q^{2}\right)=\left(4 / P^{2}\right)\left[G_{E}{ }^{2}-\left(q^{2} / 4 M^{2}\right) G_{M}{ }^{2}\right] .
\end{aligned}
$$

The symbols used above are deqned as follows: $\alpha$ is the fine structure constant $1 / 137, p_{+}\left(p_{-}\right)$is the 4-momentum of the positron (electron), $E_{+}\left(E_{-}\right)$is the energy of the positron (electron), $K$ is the energy of the incident $\gamma$ ray, $Q$ is the 4-momentum of the nucleus before interaction, $Q^{\prime}$ is the 4-momentum of the nucleus after interaction, $q$ is the momentum transfer to target nucleus $Q-Q^{\prime}, P=Q+Q^{\prime}$, and $\theta_{+}\left(\theta_{-}\right)$is the production angle of the positron (electron). 
In the kinematic region of this experiment estimates show the Compton contribution to be very small. ${ }^{5}$ For momentum transfers to the virtual photon less than the vector-meson threshold, BDF estimated the Compton term to be

$$
d \sigma_{c}=Z^{2}(E / m)^{2} \tan ^{4}(\theta / 2) d \sigma_{\mathrm{BH}},
$$

which for this experiment is $<0.01 d \sigma_{\mathrm{BH}}$. They also demonstrated that an experiment detecting the $e^{+} e^{-}$ pairs symmetrically had important advantages. First, the recoil of the target nucleus and thus corrections for form factors are minimized. Second, charge-conjugation invariance requires the interference between BetheHeitler and Compton diagrams to vanish. In principle, this interference could otherwise be as much as $10 \%$ of $d \sigma_{\mathrm{BH}}$. At symmetry, if $E$ is the energy of each lepton and $\theta$ the production angle, the momentum transfer to the virtual lepton is given by $t^{2}=-2 E^{2} \theta^{2}$, while the momentum transfer $q$ to the recoil nucleus is given by $q^{2}=-E^{2} \theta^{4}$. Thus for small angles, $\left|t^{2}\right| \gg\left|q^{2}\right|$. Under the kinematical conditions of this experiment, $|t|<400$ $\mathrm{MeV} / c$ and $\left|\left\langle q^{2}\right\rangle\right|^{1 / 2}<50 \mathrm{MeV} / c$. Because $|q|$ is small, a heavy nuclear target may be used, and for a spin-zero nucleus the Bethe-Heitler cross section is proportional to $Z^{2} G_{E}{ }^{2}\left(q^{2}\right)$. As seen from the following accurate analytical expression ${ }^{6}$ for $G_{E}$, little correction is necessary for elastic carbon form factors:

$$
G_{E}^{2}\left(q^{2}\right)=\left(1-\frac{b a^{2} q^{2}}{2(2+3 b)}\right) e^{-a^{2} q^{2} / 4},
$$

where for carbon

$$
b=\frac{4}{3} \quad \text { and } \quad a=1.635 \mathrm{~F} .
$$

Quasi-elastic and inelastic form factors are very small, and are not considered in the analysis of this experiment. However, the accuracy of this experiment as a test of QED is limited to a few percent by lack of knowledge of inelastic form factors, as well as by one's inability to calculate the Compton term accurately.

The arrangement used to measure the yield of $e^{+} e^{-}$ pairs is described in detail in the following sections. The pairs were detected by a double-arm magnetic spectrometer placed symmetrically about the incident photon beam. Fast electronics and counter techniques were used to identify the electrons in the presence of intense backgrounds of pions and low energy electrons. The measured yields were compared with yields calculated by integrating Eq. (1) over the acceptance of the spectrometer. Many checks were made to ensure that no systematic errors were present, and that all corrections had been correctly dealt with. Yields were measured for

${ }^{5}$ A. Krass, Phys. Rev. 138, B1268 (1965); S. D. Drell, in Proceedings of the International Symposium on Electron and Photon Interactions at High Energies, edited by G. Höhler et al. (Deutsche Physikalische Gesellschaft, Hamburg, 1965), Vol. 1, p. 71.

${ }^{6}$ J. H. Fregeau, Phys. Rev. 104, 225 (1956). electron (positron) momenta from 1167 to $2250 \mathrm{MeV} / \mathrm{c}$ for production angles from $4^{\circ}$ to $7^{\circ}$, and for momentum transfers $|t|$ from 109 to $389 \mathrm{MeV} / c$.

\section{EXPERIMENTAL ARRANGEMENT}

\section{A. Bremsstrahlung Beam}

The bremsstrahlung beam is produced by the striking of an internal rotating tungsten target by the DESY circulating electron beam. The average photon intensity is $3 \times 10^{10}$ equivalent quanta per second, the duty cycle is $2-4 \%$. The beam is defined by two lead collimators, $(10 \times 10)$ and $(15 \times 15) \mathrm{mm}^{2}$, and cleared of charged particles by three dipoles. The first two dipoles bend horizontally, the third vertically. Approximately $35 \mathrm{~m}$ downstream from the rotating target a $2.5 \mathrm{~g} / \mathrm{cm}^{2}$-thick carbon target ${ }^{7}$ was mounted on a calibrated optical bench. At the target position the beam spot is approximately square in shape, 2.5 by $2.5 \mathrm{~cm}$. The beam intensity is measured by a Wilsontype quantameter, which was calibrated in an external electron beam against a Faraday cup. ${ }^{8}$ The photons travel to the quantameter inside a vacuum pipe encased throughout its length by heavy concrete and lead.

The energy spectrum of the bremsstrahlung beam, i.e., the probability that a photon has an energy between $k$ and $k+d k$ for an incident electron energy $k_{\max }$, is given by

$$
f\left(k, k_{\max }\right) d k=d k T\left(k, k_{\max }\right) a_{t},
$$

where $T\left(k, k_{\max }\right)$ is the energy spectrum for a thin target, ${ }^{9}$ and where the factor $a_{t}$, determined experimentally, ${ }^{10}$ corrects for multiple scattering and energy loss in a thick target. The expressions used in the analysis of this experiment were

$$
\begin{aligned}
T\left(k, k_{\max }\right)= & {\left[R-0.925(Z / 137)^{2}+0.0555\right]^{-1}\left\{\left[1+(1-v)^{2}\right]\right.} \\
& \times\left[R-0.91 \gamma-0.925(Z / 137)^{2}\right]-(2 / 3)(1-v) \\
& \left.\times\left[R-0.1667-0.925(Z / 137)^{2}-0.647 \gamma\right]\right\} \\
a_{\iota}=-\left(1+30 / k_{\max }\right)\left[0.00082278(1-v)^{-1}\right. & \\
& +1.0540-0.42189 v \\
& \left.+1.0953 v^{2}-0.8049 v^{3}\right],
\end{aligned}
$$

where

$v=k\left(k_{\max }+m_{e}\right)^{-1}, \gamma=100 m_{e} Z^{-1 / 3}\left(k_{\max }+m_{e}\right)^{-1}(1-v)^{-1}$,

and

$$
R=\ln \left(183 Z^{-1 / 3}\right) .
$$

\footnotetext{
${ }^{7}$ Runs were taken for target thicknesses $T$ varying from 0.4 to $3.3 \mathrm{~g} / \mathrm{cm}^{2}$. The results show no evidence of a positive $T^{2} \mathrm{de}-$ pendence of the yield. The carbon was $99.9 \%$ pure.

${ }^{8}$ R. R. Wilson, Nucl. Instr. 1, 101 (1957). The calibration constant for the quantameter is $(3.35 \pm 0.10) \times 10^{18} \mathrm{MeV} / \mathrm{C}$. as given in Deutsches Elektronen-Synchrotron DESY Internal Report No. 65/2, Hamburg, 1966 (unpublished).

${ }^{9} \mathrm{H}$. W. Koch and J. W. Motz, Rev. Mod. Phys. 31, 920 (1959)

${ }^{10} \mathrm{H}$. D. Schultz, thesis, Deutsches Elektronen-Synchrotron DESY Report No. 66/16, 1966 (unpublished).
} 


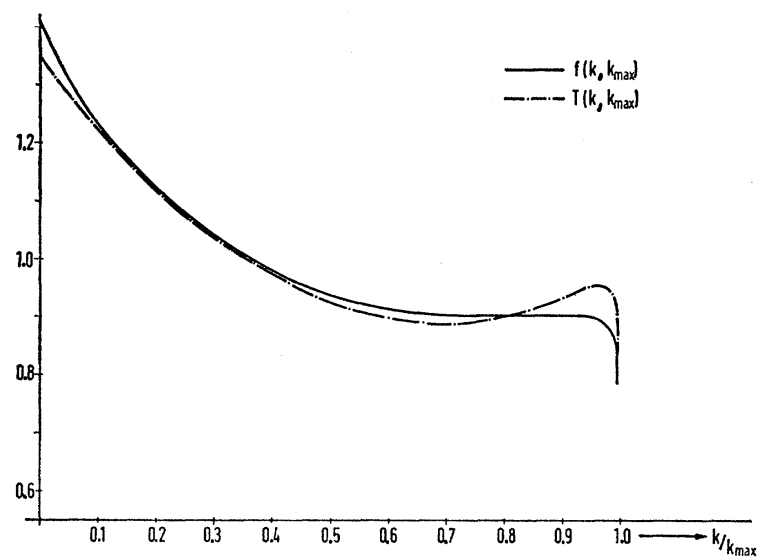

FIG. 2. Bremsstrahlung energy spectrum. $T\left(k, k_{\max }\right)$ is the thintarget spectrum. $f\left(k, k_{\max }\right)$ is the spectrum for a $0.2-\mathrm{mm}$-thick tungsten target. $k_{\max }$ is the energy of the circulating electron beam.

The quantity $m_{e}$ is the electron mass. The functions $f\left(k, k_{\max }\right)$ and $T\left(k, k_{\max }\right)$ are shown in Fig. 2.

\section{B. Spectrometer}

The spectrometer, shown in Fig. 3, consists of dipole magnets $\left(M_{D}, M_{A}, M_{B}\right)$, scintillation counters $\left(\mathrm{L}_{1}, \mathrm{~L}_{2}, \mathrm{~L}_{3}, \mathrm{~L}_{4}, \mathrm{R}_{1}, \mathrm{R}_{2}, \mathrm{R}_{3}, \mathrm{R}_{4}\right)$, shower counters (SCL,SRC), threshold Cerenkov counters (LC,RC,HL,HR), and scintillation-counter hodoscopes (TL,TR,QL,QR,VL, $\mathrm{VR}$ ). The magnet $\mathrm{M}_{\mathrm{D}}$ (maximum field $18 \mathrm{kG}$, over an effective volume $1.0 \times 1.5 \times 0.3 \mathrm{~m}^{3}$ ) separates charged particles from the $\gamma$ beam, and also sweeps very lowenergy particles out of the system. Particles with a central spectrometer momentum $p_{0}$ are bent an angle of $15^{\circ}-\theta$ by $\mathrm{M}_{\mathrm{D}}$, where $\theta$ is the horizontally projected production angle with respect to the $\gamma$ beam. To isolate the $\gamma$ beam and associated low-energy particles from the aperture of the spectrometer, much shielding is placed within the field region of $M_{D}$. However, the shielding is in all cases at least $5 \mathrm{~cm}$ away from the limiting trajectories of accepted particles, and thus is never a source of scattered background. After passing through $\mathrm{M}_{\mathrm{D}}$, the central momentum particles are bent a constant $-8^{\circ}$ by the $\mathrm{M}_{\mathrm{B}}\left(1.029 \times 0.303 \times 0.106 \mathrm{~m}^{3}\right.$ effective field volume) located $2.18 \mathrm{~m}$ downstream from the center of $\mathrm{M}_{\mathrm{D}}$. The target position and the field of $\mathbf{M}_{\mathrm{D}}$ are chosen such that the trajectory of the central

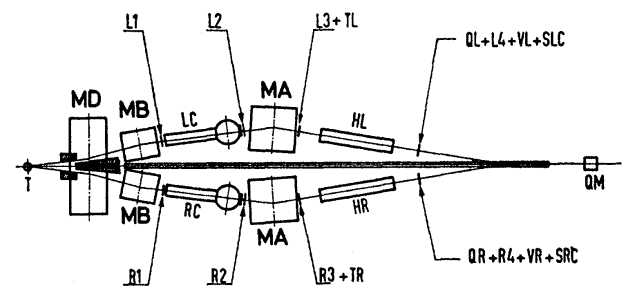

Fig. 3. Plan view of the spectrometer. ray (central momentum $p_{0}$ and angle $\theta_{0}$ ) after it enters the $\mathrm{M}_{\mathrm{B}}$ 's is identical for all settings of the spectrometer. The magnets $\mathrm{M}_{\mathrm{A}}, 5.39 \mathrm{~m}$ downstream from the $\mathrm{M}_{\mathrm{B}}$ 's, then bend the central ray a constant angle $-17.47^{\circ}$. (The $\mathrm{M}_{\mathrm{A}}$ 's have an effective field volume of $1.30 \times 0.488$ $\times 0.166 \mathrm{~m}^{3}$.) This arrangement has the following properties essential to the experiment:

(1) The acceptance of the spectrometer is not limited by the edges of magnets or by shielding, being defined instead by the scintillation trigger counters $\mathrm{L}_{2}-\mathrm{L}_{4}$, $\mathbf{R}_{\mathbf{2}}-\mathbf{R}_{\mathbf{4}}$. All counters are located such that their surfaces are not directly exposed to the target. The instantaneous rate in $\mathrm{L}_{2}$ and $\mathrm{R}_{2}$, the "hottest" of the triggering counters, is always $<3 \mathrm{mc} / \mathrm{sec}$; in all of the other counters, always $<100 \mathrm{kc} / \mathrm{sec}$.

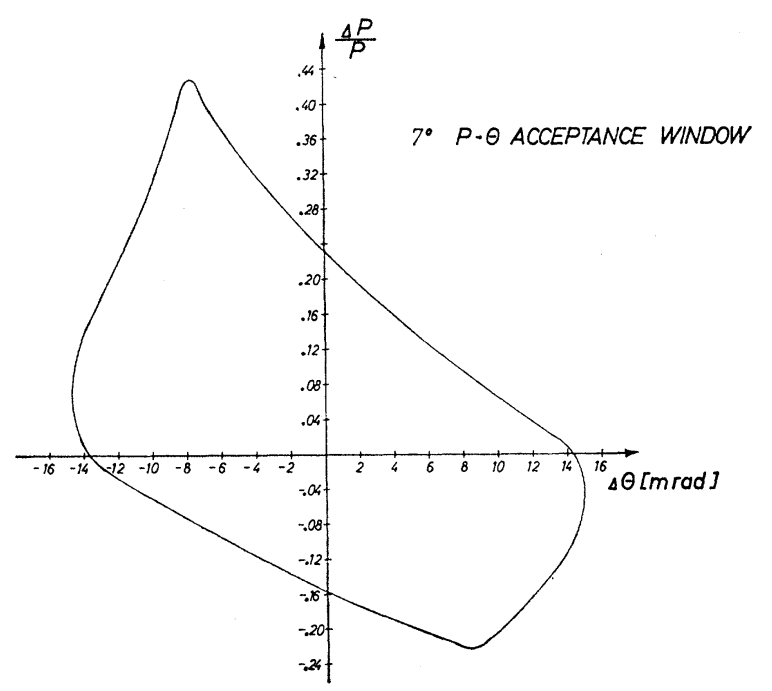

FIG. 4. Spectrometer acceptance limits. The locus of the limiting trajectories is shown as a function of $\Delta p / p$ and $\Delta \theta$ as defined in the text. The central spectrometer angle $\theta_{0}$ is $7^{\circ}$.

(2) The spread in position and angle of the particles as they pass through all the threshold and shower counters is nearly independent of the spectrometer setting. Therefore, any slight inefficiency of these counters cannot lead to a momentum-transfer-dependent effect.

(3) The spectrometer recombines rays of constant $p \theta \simeq|t|$ and therefore has a large acceptance and at the same time a good $|t|$ resolution. For a given spectrometer setting, the acceptance is $\delta p / p \simeq \pm 0.18$, $\delta \theta / \theta \simeq \pm 0.14, \delta t / \simeq \pm 0.10$, and $\delta \psi \simeq \pm 8 \mathrm{mrad}$, where $\psi$ is the projected vertical production angle. The acceptance in the invariant pair mass $m$ is $\delta m / m \simeq 0.10$.

Figure 4 shows a typical $\theta-p$ acceptance window for one of the spectrometer arms, for $\theta_{0}=7^{\circ}$. The curve shows the limiting trajectories.

Using the hodoscope counters we are able to obtain 

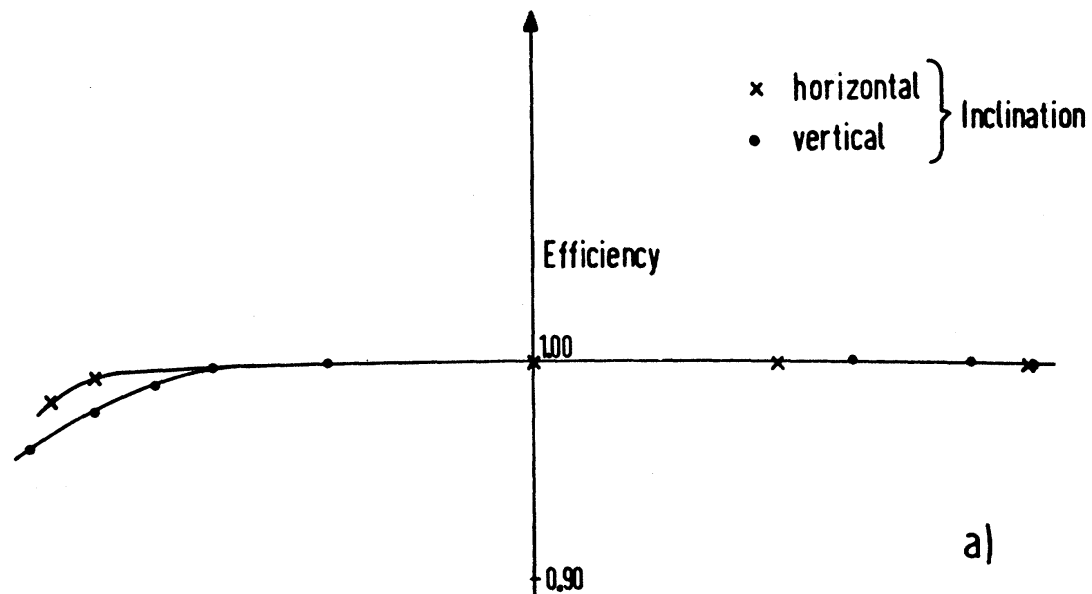

FIG. 5. (a) Efficiency of threshold counter $L C$. The effciency is shown as a function of the horizontal and vertical angle made by $1-\mathrm{GeV}$ electrons with respect to the central axis of the counter. (b) Relative $e^{+} e^{-}$ pair rate as a function of the factor by which the phototube pulse from $S R C$ was attenuated. The spectrometer setting was $\theta_{0}=4^{\circ}, p_{0}=1579 \mathrm{MeV} / c$. The arrow shows the operating point used for all runs in the experiment for which $p_{0}=1.579$ $\mathrm{MeV} / c$.
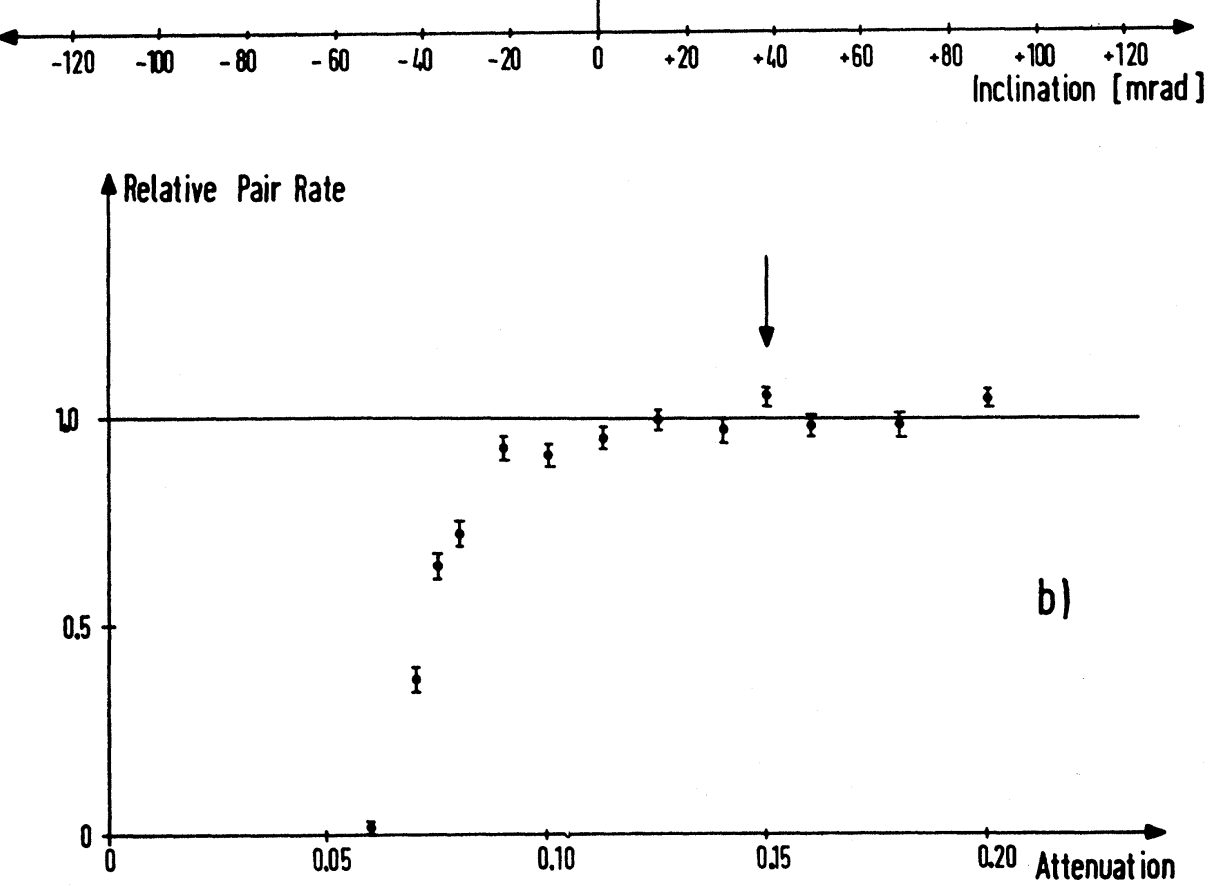

resolutions $\Delta p / p= \pm 0.02, \Delta \theta / \theta= \pm 0.03, \Delta t / t=\Delta m / m$ $=0.02$, and $\Delta \psi= \pm 2 \mathrm{mrad}$.

The hodoscopes, designed primarily for a concurrent experiment to measure the photoproduction of $\rho_{0}$ mesons, were used to check the properties of the spectrometer as described later.

\section{Properties of Counters}

(1) Trigger counters. The trigger counters, only 0.3$\mathrm{cm}$ thick to minimize bremsstrahlung and multiple scattering losses, were made of Pilot-Y scintillator. Twisted lucite strips conducted the light to RCA 7746 phototubes specially selected for high gain and low noise. The pulse height is uniform over the counter area, a property necessary for fast timing. The dimensions of the counters are (in $\mathrm{cm}): \mathrm{L}_{1}, \mathrm{R}_{1}(32.0 \times 9.9)$; $\mathrm{L}_{2}, \mathrm{R}_{2}(33.0 \times 13.7) ; \mathrm{L}_{3}, \mathrm{R}_{3}(33.0 \times 14.9) ;$ and $\mathrm{L}_{4}, \mathrm{R}_{\mathbf{4}}$ (43.1 $\times 18.1) . L_{1}$ and $R_{1}$ were not used in the triggering logic and served only to monitor pions. The efficiency of each of these counters for $1-\mathrm{GeV}$ electrons was measured to be $>99.9 \%$.

(2) Cerenkov counters. The counters LC and RC, 40 $\mathrm{cm}$ in diam. and $2.5 \mathrm{~m}$ long, use 1.09 atm $\mathrm{CO}_{2}$ as radiator. An aluminized-Mylar plane mirror, onto which 


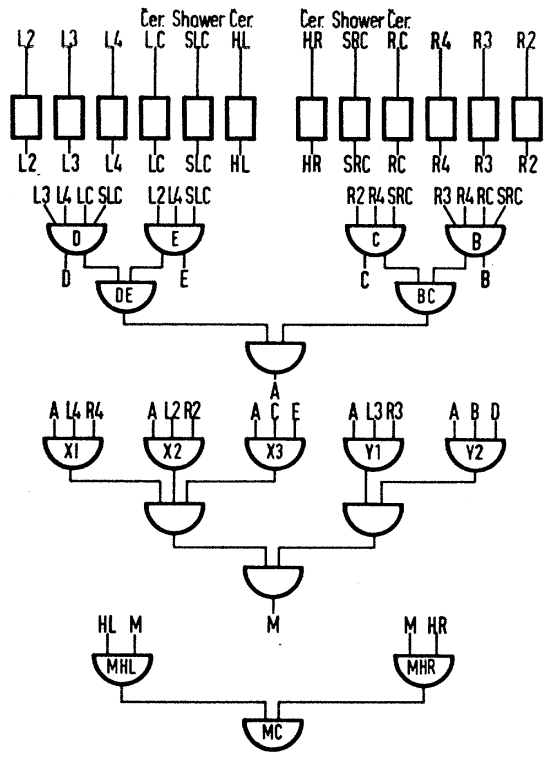

is evaporated thorium fluoride, reflects the light upwards to a 58 UVP phototube located at the focus of a parabolic mirror. The counters HL and HR, wooden boxes $40 \mathrm{~cm}$ high, $55 \mathrm{~cm}$ wide, and $3 \mathrm{~m}$ long, have 1-atm freon-12 as radiator. A concave mirror at the downstream end directs the Čerenkov light onto three 58 AVP phototubes, the pulses from which are added linearly. The shower counters SLC, SRC each consist of 16 sheets of UVT Lucite $(55 \mathrm{~cm}$ wide, $30 \mathrm{~cm}$ high, and $0.63 \mathrm{~cm}$ thick) interspersed with 16 lead sheets each 1 radiation length thick. For a description of the properties of such shower counters, see Ref. 11.

The Cerenkov counters were also tested in a $1-\mathrm{GeV}$ electron beam. In Fig. 5(a) is shown the efficiency of LC as a function of the angle of the electron beam with respect to the central axis of the counter. For the angular divergences encountered in the experiment $(\leq 50$ mrad) the efficiency is $>99 \%$. The other Cerenkov counters $\mathrm{RC}, \mathrm{HL}$, and $\mathrm{HR}$ were also $>99 \%$ efficient. The shower counters SLC and SRC were $>99.8 \%$ efficient over their areas. The operating point of each shower counter was chosen on the basis of curves such as Fig. 5(b), taken for each spectrometer momentum setting $p_{0}$.

During the experiment the pion rejection of the threshold and shower counters was constantly monitored. Even under the highest instantaneous rates encountered, the combined rejection of the pair (LC, RC) was always $>4 \times 10^{3}$; of (HL,HR), always $>1 \times 10^{3}$; and of (SLC,SRC), always $>100$.

Muons as well as pions were rejected by the threshold and shower counters. In the counters LC and RC the muon threshold was $3.4 \mathrm{GeV} / c$, roughly $500 \mathrm{MeV}$ above

${ }^{11}$ C. A. Heusch and C. Y. Prescott, IEEE Trans. Nucl. Sci. 4, 213 (1965). the high-momentum limit of the single-arm spectrometer acceptance. The shower counters were only $\simeq 1 \%$ efficient on muons, as measured with cosmic rays. Muon contamination (including that from $\pi \rightarrow \mu$ decay) is estimated to be $<0.1 \%$.

\section{Electronics}

The electronics system shown in Fig. 6 processed the counter pulses. Logic circuits capable of operating at $125 \mathrm{Mc} / \mathrm{sec}$ were used to minimize dead time and accidentals. The triggering requirements were first (circuits B, C, D, E) that LC, RC, SLC, and SRC give pulses in coincidence with the acceptance-defining scintillator pulses. Then the circuits $\mathrm{BC}$ and DE selected electrons (positrons) which had been accepted by one spectrome-

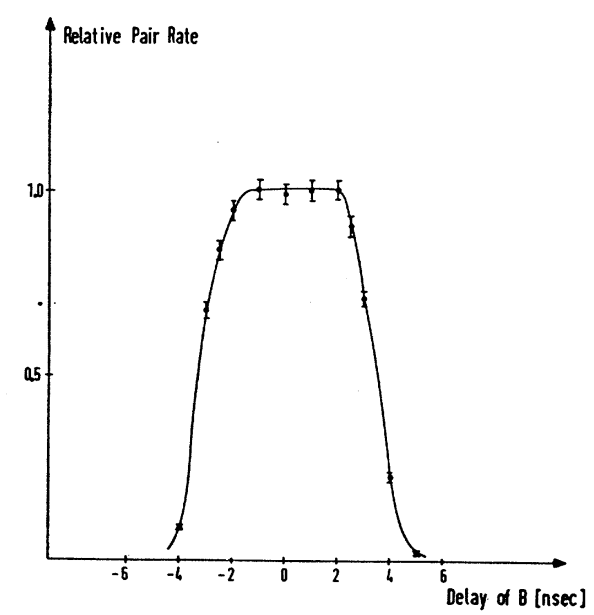

FIG. 7. Typical delay curve. The rate of the circuit $Y_{2}$ is shown as a function of the delay of the circuit $B$. (see Fig. 4). 
FIG. 8. Curve A is the sixfold differential cross section $d \sigma_{\mathrm{BH}}$ as a function of $\theta_{-}$, with the other variables held fixed at the center of the spectrometer acceptance, namely, $E_{+}=E_{-}=2.250 \mathrm{GeV}, \theta_{+}=\theta_{-}=7^{\circ}$, and $\phi_{+}=180^{\circ}$. Curve $B$ is the averaged differential cross section $d^{2} \sigma / d \phi_{+} d \phi_{-}$(see text for definition) for the same spectrometer setting, with again $\phi_{+}=180^{\circ}$.

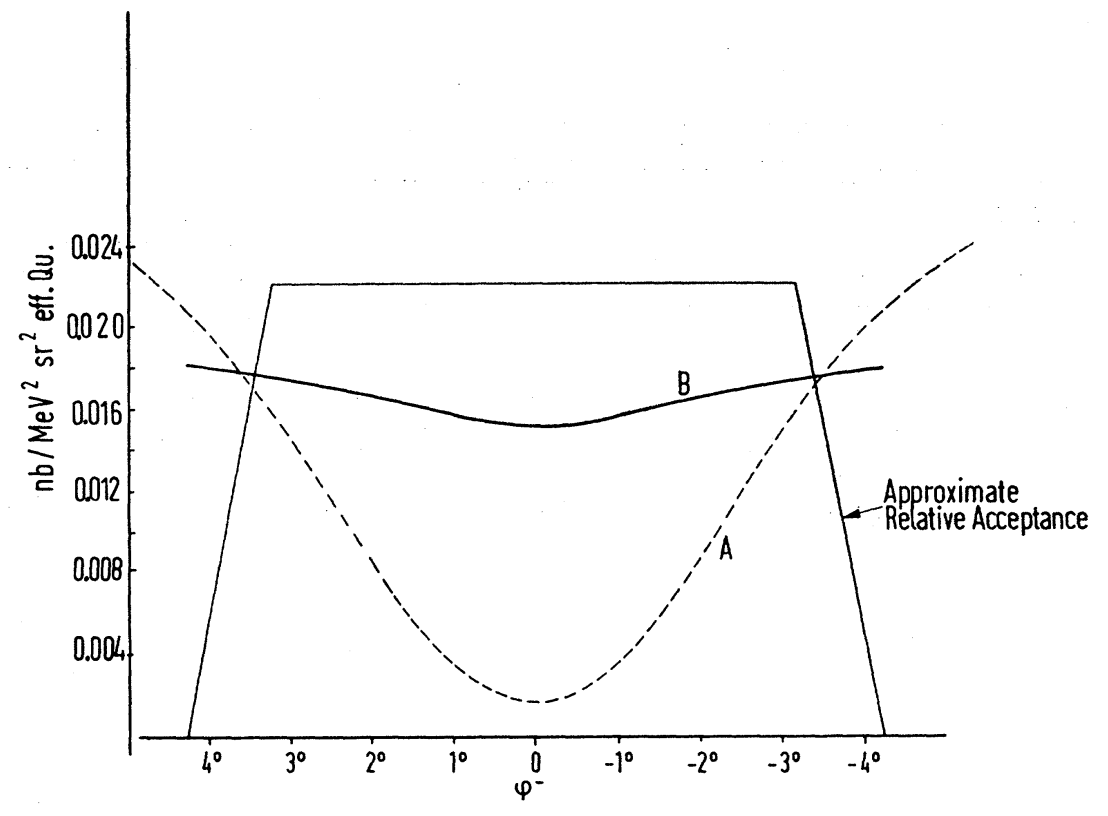

ter arm. The coincidence $A, 8 \mathrm{nsec}$ wide, then gave a preliminary indication that an electron-positron pair had been accepted. A 10-nsec-long output pulse from $A$ was used as a gate for the even more stringent triggering requirements $\mathrm{X}_{1}, \mathrm{X}_{2}, \mathrm{X}_{3}, \mathrm{Y}_{1}$, and $\mathrm{Y}_{2}$, whose resolutions were $8,7,6,5$, and 4 nsec, respectively. Finally, the coincidence $M$ between these five circuits defined an $e^{+} e^{-}$pair event. As an independent check, the left-right-arm logic system (shown at the right of the figure) with $100-\mathrm{mc} / \mathrm{sec}$ circuitry monitored the total (pion and electron) pair rate $\left(\mathrm{Z}_{0}\right)$, the operating condition of the shower counters $\left(Z_{1}\right)$ and that of the threshold counters $\mathrm{LC}$ and $\mathrm{RC}\left(\mathrm{Z}_{2}\right)$. The $e^{+} e^{-}$pair rate was also counted by $\mathrm{Z}_{1} \mathrm{Z}_{2}$, a 12 -nsec-wide coincidence between $Z_{1}$ and $Z_{2}$, and by $Z_{3} Z_{4}$, an independent 16nsec-wide coincidence between the two arms. The circuitry $V_{0}, V_{1}, V_{2}$, etc., similar to that just described except that pulses are purposely mistimed by $50 \mathrm{nsec}$, was used to monitor randoms at all points in the system. Figure 7 shows a typical delay curve obtained during the experiment. The counting rate of the circuit $Y_{2}$ is shown as a function of the delay of the pulses from the circuit B.

The logic was designed and operated to minimize and monitor the following corrections to the master rate $M$ :

(1) Random accidentals. Because the single-arm electron rate was as much as 2000 times higher than the electron-pair rate, the contribution of random coincidences had to be monitored and kept lower than a few percent. For this reason, the incident-beam intensity was always kept low enough so that the rates of circuits $\mathrm{X}_{1}, \mathrm{X}_{2}, \mathrm{X}_{2}, \mathrm{Y}_{1}, \mathrm{Y}_{2}$, and $\mathrm{Z}_{1} \mathrm{Z}_{2}$ never exceeded the master rate $M$ by more than $5 \%$. Normally, for $(2-3) \times 10^{10}$ equivalent quanta/sec incident photon intensity the rates differed by $<1 \%$. Therefore, the random accidentals in the rate $M$ are $<1 \%$, and can be calculated from the relative rates of $\mathrm{X}_{1}, \mathrm{X}_{2}, \mathrm{X}_{3}, \mathrm{Y}_{1}, \mathrm{Y}_{2}$, and $\mathrm{Z}_{1} \mathrm{Z}_{2}$.

(2) Pion contamination. The ratio of pion pairs to electron pairs was in some cases as high as 1000 to 1 . Although the requirements for $M$ provide a pion rejection $>10^{5}$, we used the counters $\mathrm{HL}$ and $\mathrm{HR}$ to monitor the adequacy of this rejection. Coincidences MHL (MHR) were made between $\mathrm{M}$ and $\mathrm{HL}$ (HR), and finally the coincidence $\mathrm{MC}$ was made between MHL and MHR. The rates of M, MHL, MHR, and MC all agreed to within $1 \%$ throughout the experiment. We are thus certain that the data are not contaminated with pions; if they were, the additional pion rejection of $\mathrm{HL}$ and $\mathrm{HR}$ would cause the rates MHL, MHR, and $\mathrm{MC}$ to be significantly lower than $\mathrm{M}$.

(3) Dead time. We monitored each circuit with a fast scaler, and found that the total dead time of the system was normally $2-3 \%$, and never more than $5 \%$.

The stability of all counting rates was constantly checked during the experiment, and all counter voltages were kept constant to within $\pm 10 \mathrm{~V}$. The left-arm rate agreed with the right-arm rate to within a few percent, and the reproducibility of the $e^{+} e^{-}$rate was better than $3 \%$ over a two-month running period.

\section{ANALYSIS}

The $e^{+} e^{-}$yield predicted by QED was obtained by integrating the product $f\left(k, k_{\max }\right) d \sigma_{\mathrm{BH}}$ over the spectrometer acceptance and target position. The thicktarget bremsstrahlung energy spectrum $f\left(k, k_{\max }\right)$ is given by Eq. (4) and $d \sigma_{\mathrm{BH}}$ is given by Eq. (1). For pairs produced at exact symmetry, the function $d \sigma_{\mathrm{BH}}$ exhibits a sharp dip, as shown in Fig. 8, curve A. While care 
must be exercised in averaging this cross section, it should be pointed out that in practice (that is, for acceptances consistent with reasonable rates) the total $e^{+} e^{-}$yield is relatively insensitive to the presence of the dip. This is illustrated by curve B of Fig. 8 , where the function

$$
\frac{d^{2} \sigma}{d \boldsymbol{\phi}_{+} d \boldsymbol{\phi}_{-}}=\frac{\boldsymbol{\int} d \sigma_{\mathrm{BH}} A\left(E_{+}, \theta_{+}, \phi_{+}, E_{-}, \theta_{-}, \phi_{-}\right) d E_{+} d E_{-} d \theta_{+} d \theta_{-}}{\boldsymbol{\int} A\left(E_{+}, \theta_{+}, \phi_{+}, E_{-}, \theta_{-}, \phi_{-}\right) d E_{+} d E_{-} d \theta_{+} d \theta_{-}}
$$

( $A$ is the spectrometer acceptance function for a pair produced at the center of the target), shows only slight evidence of the dip. Curves obtained by averaging $d \sigma_{\mathrm{BH}}$ over other combinations of four variables show even less evidence of the dip. In fact, the value of $d \sigma_{\mathrm{BH}}$ averaged over all six variables changes but $5 \%$ when the $\phi$ acceptance range is doubled and thus does not depend significantly upon the spectrometer acceptance limits.

The theoretical $e^{+} e^{-}$yields were calculated by two independent methods. The first was a conventional Monte Carlo technique whereby events with randomly generated production angles, momenta, and target positions were exposed to the magnetic fields and aperture of the system. In this manner the average cross section, the spectrometer acceptance, and various kinematical quantities were simultaneously calculated. Essential to the Monte Carlo integration was the accurate determination of the magnet-transport equations. Because of the large momentum range accepted, neither first- nor second-order transport theory could be used. Instead, the equations were determined by numerically integrating a family of 40 trajectories through a grid of the measured field values of each magnet (these fields were known to 3 parts in $10^{4}$ ). The transport coefficients were then obtained from the trajectories by a least-squares method. The transport equations included all terms linear, bilinear, and pure quadratic in $x, x^{\prime}, z, z^{\prime}$, and $\delta p / p$ (except those excluded by symmetry) and terms up to fourth order in $(\delta p / p) /$ $(1+\delta p / p)$. Comparing these coefficients with those obtained from first- and second-order theory, we found good agreement, any differences being attributable to the greater accuracy of the fourth-order calculation. A sufficient number of events were treated by the Monte Carlo technique to determine the theoretical yield within an uncertainty of $\pm 2.5 \%$. The effects of multiple scattering and bremsstrahlung loss in the target and along the spectrometer were also considered. For each of the absorbing media a multiple-scattering angle was generated at random from a normal distribution with the variance $^{12}$

$$
\theta_{\mathrm{s}}{ }^{2}=(21 / \beta p)^{2} L(1+\epsilon),
$$

${ }_{12}$ CERN Users Handbook, CERN, Geneva, 1962 (unpublished). ${ }^{13}$ B. Rossi, High Energy Particles (Prentice-Hall, Inc., Englewood Cliffs, New Jersey, 1952). where $L$ is the thickness of the material in radiation lengths, and where $\epsilon$, which ranges from 0 to -0.08 , depends on $\beta$ and the scattering material. The azimuthal scattering angle $\phi_{s}$ is generated uniformly over the range $0-2 \pi$. The projected scattering angles are then $\left(\theta_{s}\right)_{x}=\theta_{s} \cos \phi_{s},\left(\theta_{s}\right)_{y}=\theta_{s} \sin \phi_{s}$. The projected $x_{s}$ and $y_{s}$ associated with the scattering can then be generated from two normal distributions ${ }^{13}$ centered about the respective mean values $\left\langle x_{s}\right\rangle=d / 2\left(\theta_{s}\right)_{x}$ and $\left\langle y_{s}\right\rangle=d / 2$ $\left(\theta_{s}\right)_{y}$, where $d$ is the thickness in centimeters of the scattering material. It was found, however, sufficiently accurate to use the mean values themselves for the diaplacement associated with a particular scattering. In this way a significant reduction in computer time was possible (a factor of 3 ), with no detectable difference from the more rigorous treatment. The size of the multiple-scattering correction was determined accurately at several spectrometer settings; for example, at $\theta_{0}=4^{\circ}, p_{0}=1167 \mathrm{MeV} / c$ (the worst case), multiple scattering decreased the theoretical yield by 12.0 $\pm 0.5 \%$; at $\theta_{0}=7^{\circ}, p_{0}=2250 \mathrm{MeV} / c$, by $2.0 \pm 1.0 \%$.

Energy losses due to bremsstrahlung were generated in the target and along the spectrometer according to the density function ${ }^{13}$

$$
W\left(E_{0}, E, L\right)=\left[1 / E_{0}(L / \ln 2)\right]\left[\ln \left(E_{0} / E\right)\right]^{(L / \ln 2-1)},
$$

where $E_{0}$ and $E$ are the electron energies before and after bremsstrahlung, respectively. The yield obtained including this correction was compared with the yield without the correction to determine the size of the effect. The theoretical yield with bremsstrahlung included in the calculation was between 32 and $38 \%$ lower than that without bremsstrahlung. In both these yields the effect of multiple scattering was not considered, in order to save computer time. However, it was checked that no error resulted from treating multiple scattering and bremsstrahlung separately: For several spectrometer settings, the multiple scattering and bremsstrahlung were calculated simultaneously; the yield calculated in this way agreed with that obtained when multiple scattering and bremsstrahlung were treated separately.

In the second method by which the yield was calculated, each magnet was assumed to have a uniform field over its effective length; ray tracing was then used to determine the acceptance-defining trajectories. An accurate approximation ${ }^{14}$ to $d \sigma_{\mathrm{BH}}$ was then numerically integrated over the acceptance window so obtained. The acceptance and average cross sections agreed in all cases with those of the Monte Carlo calculation to within $5 \%$. As a further check, we averaged the exact cross section as given by Drell and Walecka ${ }^{15}$ over the spectrometer acceptance, finding agreement with BDF to better than $1 \%$.

\footnotetext{
${ }^{14}$ For the approximate expression for $d \sigma_{\mathrm{BH}}$, see Ref. 1.

${ }^{15}$ S. D. Drell and J. D. Walecka, Ann. Phys. (N. Y.) 28, 18
} 
Radiative corrections to the pair-production cross section were of two classes: One includes all graphs having an additional internal photon line; the other consists of processes where a real unobserved photon is emitted. In this experiment a bremsstrahlung spectrum of photons was used; contributions to the observed counting rate were possible even for processes where a hard photon of energy as large as $3.5 \mathrm{GeV}$ was emitted. Therefore, much care was taken to calculate the radiative corrections for our particular experimental arrangement. ${ }^{16}$ It was found that when the ratio $k / k_{\max }$ was kept constant the value of the radiative correction was independent of the spectrometer setting. The effect of all radiative corrections was to decrease the theoretical yield by $3.0 \pm 1.0 \%$.

\section{CONSISTENCY CHECKS}

Many experimental checks were made to ensure that the spectrometer behaved as designed:

(1) We found that the experimental yield at low momentum transfer agreed with the prediction of QED, as shown in Sec. VII, where the results are given.

(2) Wire-orbit measurements performed on both spectrometer arms agreed with the calculated central angles and momenta to within $0.5 \%$, the accuracy limit of the wire-orbit technique.

(3) To test our treatment of multiple scattering and bremsstrahlung, we placed in each arm of the spectrometer (in front of $\mathrm{L}_{2}$ and $\mathrm{R}_{2}$ ) a 0.63 -cm-thick Lucite sheet large enough to cover the spectrometer aperture. For $p_{0}=2.250 \mathrm{GeV} / c, \theta_{0}=4^{\circ}$, a $(20 \pm 5) \%$ decrease in the yield was observed, in good agreement with the calculated decrease of $(18 \pm 1) \%$.

(4) At a production angle of $4^{\circ}$ and a maximum bremsstrahlung energy $k_{\max }=3.05 \mathrm{GeV}$, the $e^{+} e^{-}$yield was measured as a function of the spectrometer momentum setting for $p_{0}=1.500,1.750$, and $2.000 \mathrm{GeV} / c$.

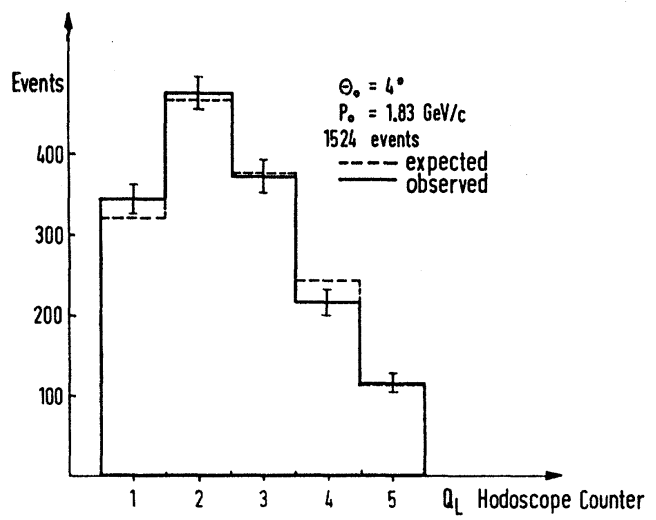

Fig. 9. Comparison of calculated and observed event distributions in the $Q_{L}$ hodoscope counters, for central spectrometer angle $\theta_{0}=4^{\circ}$ and momentum $p_{0}=1.83 \mathrm{GeV} / c$.

At the upper end of this momentum range, where the bremsstrahlung spectrum cuts off, the yield depends very sensitively upon the spectrometer acceptance. The ratios of the yields at $p_{0}=1.500,1.750$, and $2.000 \mathrm{GeV} / c$ to the yield at $p_{0}=1.167 \mathrm{GeV} / c$ were, respectively: (a) experiment: $0.305 \pm 0.032 ; 0.025$ $\pm 0.007 ;<0.001$; (b) theory: $0.351 ; 0.0370 ; 0$. The consistency between experimental and theoretical yields demonstrated that the acceptance had been accurately calculated.

(5) To check the properties of the spectrometer and of the programs used, the distributions of electron-pair events were measured by the hodoscopes. In a typical data run, with central angle $4^{\circ}$, central momentum $1.830 \mathrm{GeV} / c$, the distribution shown in Fig. 9 was obtained in the hodoscope QL, the expected and observed distributions being in good agreement. Similar results were obtained for the other hodoscopes.

(6) For the spectrometer setting $p_{0}=2.250, \theta_{0}=4^{\circ}$, the hodoscope data was compiled to give a distribution

FIg. 10. Distribution of invariant mass for the spectrometer setting $\theta_{0}=4^{\circ}, p_{0}=2.250 \mathrm{GeV} / c$. The histogram shows the observed number of events, the smooth curve shows the theoretical yield. The theoretical curve has been multiplied by 0.94 to be normalized to the experimental yield. Part of this data was accumulated in subsequent experiments.

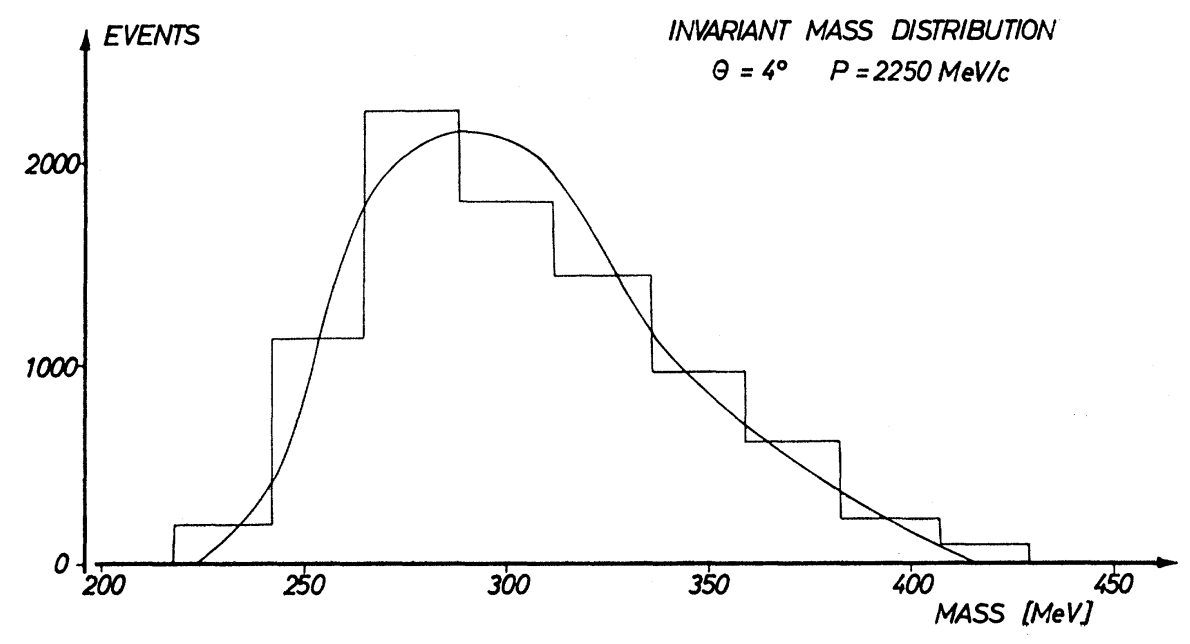

${ }^{16} \mathrm{~S}$. J. Brodsky (to be published). 


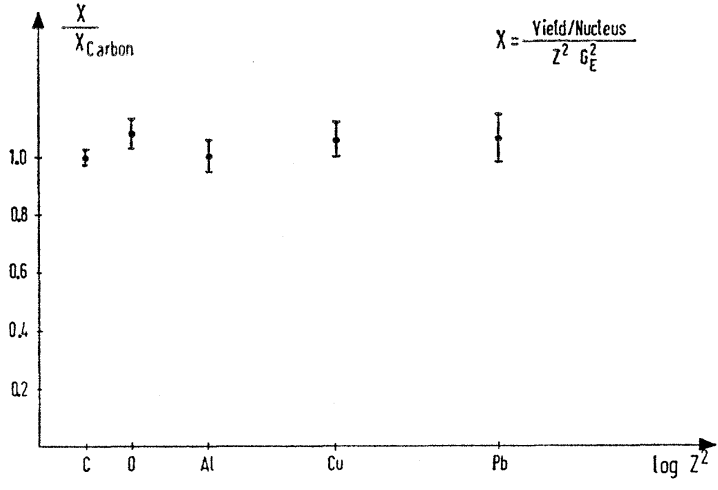

FIG. 11. Pair production for various target materials, at a spectrometer setting of $\theta_{0}=4^{\circ}, p_{0}=2.250 \mathrm{GeV} / c$.

of the invariant mass of the pair. As shown in Fig. 10 the result is consistent with the calculated spectrum.

(7) The electron-pair rate did not change when the shielding inside the gap of $\mathrm{M}_{\mathrm{D}}$ was moved either toward or away from the accepted region. This is good evidence that the shielding is not a source of background events. Checks (3), (4), and (5) above indicate that scattering from $\mathrm{M}_{\mathrm{B}}$ and $\mathrm{M}_{\mathrm{A}}$ pole faces also did not contribute to the measured yield.

(8) To monitor the quantameter sensitivity, we placed in front of the quantameter a 1 -mm-thick $\mathrm{Cu}$ plate, which was viewed at an angle of $30^{\circ}$ by a fivecounter telescope. A 2-mm-thick Al plate and a 1.8 $\mathrm{kG} f \mathrm{ft}$ permanent magnet were put in front of the telescope to clear low-energy particles. Throughout the experiment the ratio of quantameter charge to the number of counts in the telescope was constant to within $\pm 5 \%$.

(9) To check that the nucleus recoils coherently, the $e^{+} e^{-}$yields were measured to $3 \%$ accuracy with $\mathrm{C}, \mathrm{O}$,

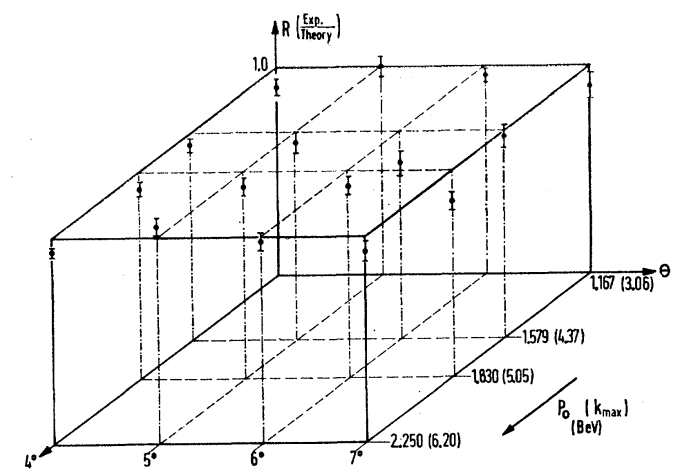

FIG. 12. The ratio of experiment to theory is shown for all data points. The points are arranged in a matrix, the study of which shows (see text) that systematic errors in the experiment are insignificant.

$\mathrm{Al}, \mathrm{Cu}$, and $\mathrm{Pb}$ targets for a momentum transfer to the nucleus $\left|\left\langle q^{2}\right\rangle\right|^{1 / 2}=30 \mathrm{MeV} / c$. The relative yields are shown in Fig. 11. Good agreement is seen with the law $Z^{2} G_{E}{ }^{2}\left(q^{2}\right)$, where $G_{E}{ }^{2}\left(q^{2}\right)$ is the electric form factor of the target nucleus and $Z$ the nuclear charge.

\section{PROCEDURE}

(1) The yield was measured at 16 settings of the spectrometer, at least 400 events being accumulated for each. At most settings 800 events were accumulated.

(2) At each setting half the events were taken at each polarity of the spectrometer. Thus interference between Compton and Bethe-Heitler processes does not contribute.

(3) Approximately $25 \%$ of the running time at each setting was used to measure target-out rates. These rates were found to be nearly independent of angle and momentum, never exceeding $10 \%$ of the rate with target in place.

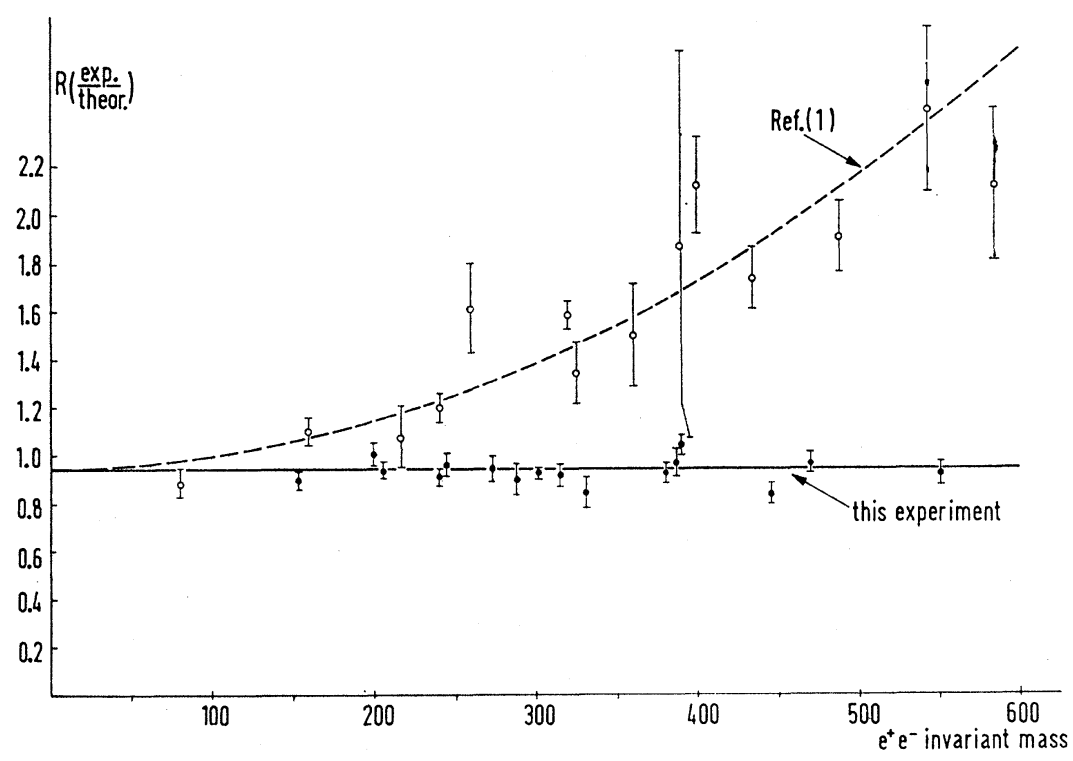

FIG. 13. The ratio of experiment to theory is shown as a function of the $e^{+} e^{-}$ invariant mass. The straight line is the best fit to our data. Earlier work at CEA (Blumenthal et al., Ref. 1) is also shown. To facilitate comparison the normalization of the CEA data is changed in the figure so that the best-fit curve agrees with our best fit at zero pair mass. 
TABLE I. Data and results.

\begin{tabular}{|c|c|c|c|c|c|c|c|c|c|c|c|c|c|}
\hline \multicolumn{4}{|c|}{ Spectrometer setting } & \multirow[t]{2}{*}{$\begin{array}{l}\text { Number } \\
\text { of events }\end{array}$} & \multicolumn{3}{|c|}{$\begin{array}{c}\text { Experimental yield } \\
{\left[\text { events } /\left(10^{12} \text { equivalent }\right.\right.} \\
\left.\text { quanta })\left(\mathrm{g} / \mathrm{cm}^{2}\right)\right]\end{array}$} & \multicolumn{4}{|c|}{ Corrections $^{\mathrm{a}}$} & \multicolumn{2}{|c|}{$\begin{array}{c}\text { Theoretical } \\
\text { yield }\end{array}$} \\
\hline & $\begin{array}{c}P_{0} \\
(\mathrm{GeV} / c)\end{array}$ & $\begin{array}{c}\left|\left\langle t^{2}\right\rangle\right|^{1 / 2} \\
(\mathrm{MeV})\end{array}$ & $\begin{array}{c}\left|\left\langle q^{2}\right\rangle\right|^{1 / 2} \\
(\mathrm{MeV})\end{array}$ & & $\underset{\text { in }}{\text { Target }}$ & $\begin{array}{c}\text { Target } \\
\text { out }\end{array}$ & Net & $\begin{array}{l}\text { Dead } \\
\text { time } \\
\text { and } \\
\text { acc. }\end{array}$ & $\begin{array}{l}\text { Radi- } \\
\text { ative } \\
\text { correc- } \\
\text { tions }\end{array}$ & $\begin{array}{c}\text { Brems- } \\
\text { strah- } \\
\text { lung }\end{array}$ & $\begin{array}{l}\text { Total } \\
\text { correction } \\
\text { factor }\end{array}$ & & \\
\hline $4^{\circ}$ & $\begin{array}{l}1.167 \\
1.579 \\
1.830 \\
2.250\end{array}$ & $\begin{array}{l}109 \\
149 \\
170 \\
214\end{array}$ & $\begin{array}{l}15.1 \\
20.7 \\
23.0 \\
28.5\end{array}$ & $\begin{array}{r}800 \\
1401 \\
1002 \\
3004\end{array}$ & $\begin{array}{l}9.740 \\
5.557 \\
4.299 \\
2.719\end{array}$ & $\begin{array}{l}0.794 \\
0.392 \\
0.338 \\
0.153\end{array}$ & $\begin{array}{l}8.946 \\
5.165 \\
3.961 \\
2.566\end{array}$ & $\begin{array}{l}0.958 \\
0.963 \\
0.990 \\
0.998\end{array}$ & 1.030 & 1.616 & $\begin{array}{l}1.595 \pm 0.021 \\
1.603 \pm 0.021 \\
1.648 \pm 0.020 \\
1.661 \pm 0.019\end{array}$ & $\begin{array}{c}15.90 \\
8.869 \\
7.156 \\
4.593\end{array}$ & $\begin{array}{l}0.897 \pm 0.038 \\
0.934 \pm 0.031 \\
0.912 \pm 0.034 \\
0.928 \pm 0.022\end{array}$ \\
\hline $5^{\circ}$ & $\begin{array}{l}1.167 \\
1.579 \\
1.830 \\
2.250\end{array}$ & $\begin{array}{l}141 \\
192 \\
223 \\
275\end{array}$ & $\begin{array}{l}19.5 \\
26.5 \\
30.0 \\
36.4\end{array}$ & $\begin{array}{l}800 \\
619 \\
800 \\
969\end{array}$ & $\begin{array}{l}2.867 \\
1.468 \\
1.128 \\
0.7868\end{array}$ & $\begin{array}{l}0.277 \\
0.139 \\
0.116 \\
0.0583\end{array}$ & $\begin{array}{l}2.590 \\
1.329 \\
1.012 \\
0.7285\end{array}$ & $\begin{array}{l}0.978 \\
0.992 \\
0.999 \\
1.001\end{array}$ & & 1.577 & $\begin{array}{l}1.588 \pm 0.021 \\
1.611 \pm 0.020 \\
1.622 \pm 0.019 \\
1.626 \pm 0.019\end{array}$ & $\begin{array}{l}4.101 \\
2.264 \\
1.792 \\
1.135\end{array}$ & $\begin{array}{l}1.003 \pm 0.045 \\
0.946 \pm 0.048 \\
0.916 \pm 0.043 \\
1.044 \pm 0.042\end{array}$ \\
\hline $6^{\circ}$ & $\begin{array}{l}1.167 \\
1.579 \\
1.830 \\
2.250\end{array}$ & $\begin{array}{l}173 \\
234 \\
269 \\
332\end{array}$ & $\begin{array}{l}24.2 \\
32.4 \\
36.3 \\
44.2\end{array}$ & $\begin{array}{l}600 \\
377 \\
600 \\
821\end{array}$ & $\begin{array}{l}0.8036 \\
0.4118 \\
0.3159 \\
0.2186\end{array}$ & $\begin{array}{l}0.0622 \\
0.0512 \\
0.0153 \\
0.0211\end{array}$ & $\begin{array}{l}0.7414 \\
0.3606 \\
0.3006 \\
0.1975\end{array}$ & $\begin{array}{l}0.985 \\
1.004 \\
1.003 \\
1.006\end{array}$ & & 1.529 & $\begin{array}{l}1.551 \pm 0.021 \\
1.581 \pm 0.019 \\
1.580 \pm 0.020 \\
1.584 \pm 0.019\end{array}$ & $\begin{array}{l}1.201 \\
0.6732 \\
0.5135 \\
0.3236\end{array}$ & $\begin{array}{l}0.957 \pm 0.047 \\
0.847 \pm 0.060 \\
0.925 \pm 0.043 \\
0.967 \pm 0.043\end{array}$ \\
\hline $7^{\circ}$ & $\begin{array}{l}1.167 \\
1.579 \\
1.830 \\
2.250\end{array}$ & $\begin{array}{l}202 \\
274 \\
315 \\
389\end{array}$ & $\begin{array}{l}28.9 \\
38.4 \\
44.1 \\
53.9\end{array}$ & $\begin{array}{l}515 \\
434 \\
627 \\
460\end{array}$ & $\begin{array}{l}0.2521 \\
0.1498 \\
0.1002 \\
0.0645\end{array}$ & $\begin{array}{l}0.0177 \\
0.0101 \\
0.0088 \\
0.00226\end{array}$ & $\begin{array}{l}0.2344 \\
0.1397 \\
0.0914 \\
0.0623\end{array}$ & $\begin{array}{l}1.002 \\
1.025 \\
0.998 \\
1.019\end{array}$ & & 1.477 & $\begin{array}{l}1.524 \pm 0.020 \\
1.559 \pm 0.020 \\
1.518 \pm 0.021 \\
1.550 \pm 0.021\end{array}$ & $\begin{array}{l}0.3975 \\
0.2245 \\
0.1660 \\
0.1042\end{array}$ & $\begin{array}{l}0.899 \pm 0.063 \\
0.970 \pm 0.056 \\
0.836 \pm 0.043 \\
0.926 \pm 0.048\end{array}$ \\
\hline
\end{tabular}

a The effect of multiple scattering is included in the theoretical yield. For fixed $\theta_{0}$ the bremsstrahlung correction is independent of $p_{0}$. The radiative correction is the same for all spectrometer settings.

(4) The ratio $k / k_{\max }$ was the same for all settings ( $k$ is the center pair energy, $k_{\max }$ the peak bremsstrahlung energy). The relative yields are then independent of radiative corrections and insensitive to bremsstrahlung losses.

(5) Data were taken at $\theta_{0}=4^{\circ}, 5^{\circ}, 6^{\circ}$, and $7^{\circ}$. For each value of $\theta_{0}$ measurements were made for $p_{0}=1.167$, $1.579,1.830$, and $2.250 \mathrm{GeV} / c$. Thus the 16 data points form a $4 \times 4$ grid in $\left(p_{0}, \theta_{0}\right)$ which has the following important properties:

(a) For fixed $\theta_{0}$, the relative yields are subject to the same spectrometer acceptance and bremsstrahlung corrections. Except for multiple-scattering corrections and possible small variations with $k_{\max }$ of the quantameter sensitivity, the yield should follow (after formfactor corrections) a simple $1 / k^{2}$ law.

(b) On the other hand, for fixed $p_{0}$ the relative yields are insensitive to multiple scattering, quantameter variations, and scattering from pole faces or shielding. The only possible systematic error could arise in the calculation of the spectrometer acceptance.

This method of collecting the data, changing only one variable at a time, would expose the existence of any systematic errors in the system.

\section{DATA}

In Table I are listed, for each spectrometer setting, the number of events, the average values of $\left|t^{2}\right|^{1 / 2}$ and $\left|q^{2}\right|^{1 / 2}$, the experimental and theoretical yields, the various correction factors, and finally the ratio of the corrected experimental yield to the yield cacluated as described in Sec. III. The experimental yields are ex- pressed in events $/\left(10^{12}\right.$ equivalent quanta $)\left(\mathrm{g} / \mathrm{cm}^{2}\right)$. For each setting, a 3\% correction for beam loss in the target has been applied to the experimental yields. Pion contamination, being $<1 \%$, is not corrected for.

\section{RESULTS AND CONCLUSIONS}

The ratio of theoretical to observed yields is shown as a function of $\left(p_{0}, \theta_{0}\right)$ in Fig. 12. The errors shown are statistical, including those introduced by the subtraction of target-out rates. Corrections have been made for dead time, accidentals, beam attenuation in the target, multiple scattering, and bremsstrahlung losses. As seen in the figure, each point is consistent with the prediction of QED. Thus we conclude that no significant systematic errors are present, and that the present form of QED correctly describes the production of $e^{+} e^{-}$pairs.

Figure 13 shows our results (as a function of the invariant mass $m=\sqrt{2} t$ of the $e^{+} e^{-}$pair) along with other published data. The best fit of our data linear in $m$ is

$$
\left.R=0.95\left[(1 \pm 0.04)-(0.4 \pm 1.1) \times 10^{-4} m\right)\right]
$$

where $m$ is expressed in $\mathrm{MeV} / c^{2}$.

For comparison with the CEA result we give the best fit quadratic in $m$, namely ${ }^{17}$

$$
R=0.94\left[(1 \pm 0.02)-(5.5 \pm 14.8) \times 10^{-8} m^{2}\right] .
$$

The uncertainty in normalization, estimated to be $5 \%$,

${ }^{17}$ For recent results concerning the production of muon pairs, see J. K. de Pagter, J. I. Friedman, G. Glass, R. C. Chase, M. Gettner, E. von Goeler, R. Weinstein, and A. M. Boyarski, Phys. Rev. Letters 17, 767 (1966). 
TABLE II. Comparison of various tests of quantum electrodynamics at large momentum transfers. The quantity $A$ is a normalization factor, $\Lambda$ a cutoff parameter. As in Ref. $h, F_{\mu}\left(t^{2}\right)=\left(1-\left|t^{2}\right| / \Lambda_{\mu}{ }^{2}\right)^{-1}, F_{e}\left(t^{2}\right)=\left(1-\left|t^{2}\right| / \Lambda_{e}^{2}\right)^{-1}$, and $1 / D^{2}=1 / \Lambda_{\mu}^{2}-1 / \Lambda_{e}^{2}$.

\begin{tabular}{|c|c|c|c|c|}
\hline \multirow[b]{2}{*}{ Experiment } & \multirow[b]{2}{*}{ Form of modification } & \multirow[b]{2}{*}{$\begin{array}{l}\text { Most likely } \\
\text { value }\end{array}$} & \multicolumn{2}{|c|}{ Cutoff limits $(\mathrm{GeV} / c)$} \\
\hline & & & $68 \%$ confidence & $95 \%$ confidence \\
\hline$e^{-}+e^{-} \rightarrow e^{-}+e^{-a}$ & $F\left(t^{2}\right)=\left(1-\left|t^{2}\right| / \Lambda^{2}\right)^{-1}$ & 6.2 & $\Lambda>1.1$ & $\Lambda>0.76$ \\
\hline$\gamma+\mathrm{C} \rightarrow e^{+}+e^{-}+\mathrm{C}^{\mathrm{b}}$ & $R=\sigma / \sigma_{\mathrm{BH}}=A\left(1+\left|t^{2}\right| / \Lambda^{2}\right)$ & 0.313 & $0.300 \leq \Lambda \leq 0.326$ & $\ldots$ \\
\hline$\gamma+\mathrm{C} \rightarrow e^{+}+e^{-}+\mathrm{C}^{\mathrm{c}}$ & $R=A\left(1+\left|t^{2}\right| / \Lambda^{2}\right)$ & 0.360 & $0.315 \leq \Lambda \leq 0.438$ & $\ldots$ \\
\hline \multirow{4}{*}{$\gamma+\mathrm{C} \rightarrow e^{+}+e^{-}+\mathrm{C}^{d}$} & $R=A\left(1-\left|t^{2}\right| / \Lambda^{2}\right)$ & 3.0 & $\Lambda>1.8$ & $\Lambda>1.3$ \\
\hline & $R=A\left(1+\left|t^{2}\right| / \Lambda^{2}\right)$ & $\infty$ & $\Lambda>2.0$ & $\Lambda>1.4$ \\
\hline & $R=A\left(1-\left|t^{4}\right| / \Lambda^{4}\right)$ & 1.1 & $\Lambda>0.82$ & $\Lambda>0.71$ \\
\hline & $R=A\left(1+\left|t^{4}\right| / \Lambda^{4}\right)$ & $\infty$ & $\Lambda>0.88$ & $\Lambda>0.73$ \\
\hline$(g-2) / 2$ of muon ${ }^{e}$ & $\left\{1-\frac{2}{3}\left(m_{\mu}^{2} / \Lambda^{2}\right)\left[\ln \left(\Lambda^{2} / m_{\mu}^{2}\right)+\frac{1}{3}\right]\right\}$ & $\ldots$ & $\Lambda>2.8$ & $\Lambda>2.0$ \\
\hline$\gamma+\mathrm{C} \rightarrow \mu^{+}+\mu^{-}+\mathrm{C}^{\mathrm{f}}$ & $R=A\left(1-2\left|t^{2}\right| / \Lambda^{2}\right)$ & $\ldots$ & $\ldots$ & $\Lambda>0.85$ \\
\hline \multirow[t]{2}{*}{$\gamma+\mathrm{C} \rightarrow \mu^{+}+\mu^{-}+\mathrm{Cg}^{\mathrm{g}}$} & $R=A\left(1-2\left|t^{2}\right| / \Lambda^{2}\right)$ & 1.2 & $\ldots$ & $0.94 \leq \Lambda \leq 2.5$ \\
\hline & $R=A\left(1-\left|t^{2}\right| / \Lambda^{2}\right)$ & 0.85 & $\ldots$ & $0.66 \leq \Lambda \leq 1.8$ \\
\hline$(\mu+p \rightarrow \mu+p) /(e+p \rightarrow e+p)^{\mathrm{h}}$ & $F_{\mu}\left(t^{2}\right) / F_{e}\left(t^{2}\right)=1+\left|t^{2} /\right| D^{2}$ & $\cdots$ & $\cdots$ & $D>2.9$ \\
\hline
\end{tabular}

a W. C. Barber, B. Richter, B. Gittleman, G. K. O'Neill, Phys. Rev. Letters 16, 1127 (1966).

b Reference 1 .

- Reference 2.

d G. Charpak, F. J. Farley, R. L. Garwin, T. Muller, J. C. Sens, and A. Zichichi, Phys. Letters 1, 16 (1962); Nuovo Cimento 37, 1241 (1965). A. Alberigi-Quaranti, M. DePretis, G. Marini, A. Odian, G. Stoppini, and L. Tau, Phys. Rev. Letters 9, 226 (1962).

h R. Cool, A. Maschke, L. M. Lederman, M. Tannenbaum, R. Ellsworth, A. Melissinos, J. W. Tinlot, and T. Yamanouchi, Phys. Rev. Letters 14, 724 (1965).

is not included in the errors given for the above two fits.

Both these fits are consistent with a straight line of zero slope. The CEA result, on the other hand, is

$$
R=0.67\left[(1 \pm 0.04)+(513 \pm 38) \times 10^{-8} m^{2}\right]
$$

which is inconsistent with our result.

It has been shown by Kroll ${ }^{18}$ that a breakdown of QED consistent with very general requirements must be of at least fourth power in $|t|$. In this spirit we have made a maximum likelihood fit of our data to the hypotheses

$$
R=A\left(1 \pm\left|t^{4}\right| / \Lambda^{4}\right),
$$

where $\Lambda$ is a cutoff parameter. The results are shown in Fig. 14.

To conclude the discussion, we list in Table II various tests of QED at large momentum transfers.

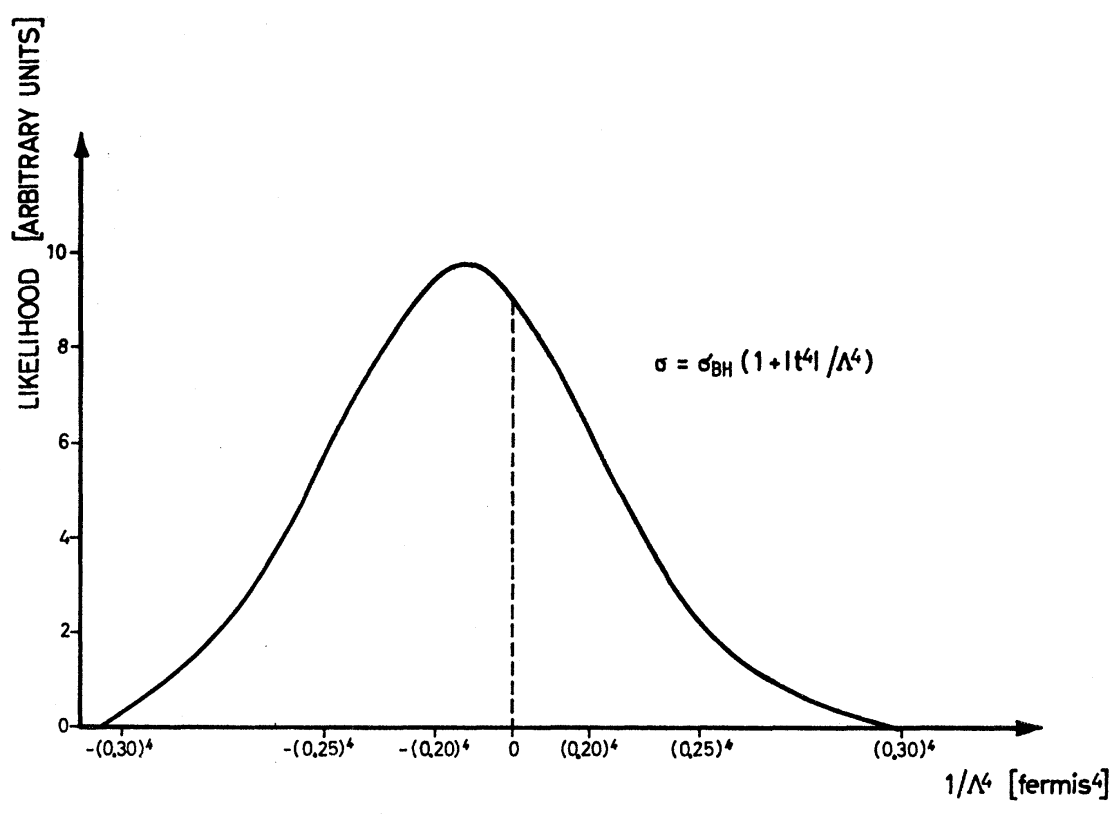

FIG. 14. Likelihood function as a function of $1 / \Lambda^{4}$ for a QED modification of the form $R=1+\left|t^{4}\right| / \Lambda^{4}$. The curve gives the likelihood for the two distinct breakdown hypotheses $R=1$ $+\left|t^{4}\right| / \Lambda^{4}$ and $R=1-\left|t^{4}\right| / \Lambda^{4}$.

${ }^{18}$ N. H. Kroll, CERN Report No, 66/430/5-Th. 647, 1966 (unpublished), 
It is seen that this experiment provides the most sensitive probe of the validity of quantum electrodynamics for electrons to date.

\section{ACKNOWLEDGMENTS}

Dr. S. J. Brodsky of Columbia University merits special thanks for his calculation of the radiative corrections to the yield. We are grateful for the enthusiastic support of Professor W. Jentschke and Professor P. Stähelin of DESY and L. M. Lederman and S. Devons of Columbia, which made this collaboration possible. We thank Dr. H. O. Wüster and Dr. D. Lublow for extend- ing to us the DESY computing facilities, and $\mathrm{H}$. Kumpfert and the synchrotron staff for their skillful operation of the machine. We acknowledge the interest of Dr. H. D.Schulz, Dr. W. Schultze, and Dr. D. Degèle in the early phases and setting up of the experiment, and the many interesting discussions with Professor $\mathrm{H}$. Joos and Professor G. Weber, and Dr. F. Gutbrod, Dr. D. Schildknecht, and Dr. E. D. Kohaupt. One of us (C. C.T.) would like to thank Professor S. D. Drell and Professor N. W. Kroll for helpful discussions regarding theoretical aspects of the experiment. Lastly, we acknowledge discussion with Professor F. M. Pipkin and Professor R. Talman. 